\title{
Molecular Dynamics Study of the Adhesion between End-grafted Polymer Films
}

\author{
Hiroshi Morita, ${ }^{1, \dagger}$ Masamichi Yamada, ${ }^{2}$ Tetsuo YAMAguchi, ${ }^{1}$ and Masao DoI ${ }^{1}$ \\ ${ }^{1}$ Japan Science and Technology Agency \& Department of Applied Physics, The University of Tokyo, \\ 7-3-1 Hongo, Bunkyo-ku, Tokyo 113-8656, Japan \\ ${ }^{2}$ Department of Computational Science and Engineering, Nagoya University, \\ Furo-cho, Chikusa-ku, Nagoya 464-8603, Japan
}

(Received May 30, 2005; Accepted July 6, 2005; Published October 15, 2005)

\begin{abstract}
Adhesion between two polymer films consisting of end grafted polymer is studied by coarse-grained molecular dynamics. Two types of polymer films are considered: one-end grafted polymer (the straight polymer) and the two-end grafted polymer (the loop polymer). The stress-distance curve for these polymers is obtained at various temperatures. It is found that (1) the separation between the films takes place by the formation of fibrils or cavities, and the adhesion is stronger in the former case than in the latter, (2) the fibrils appear in the case that the temperature is below one of the glass transition temperatures of the two films, and (3) the adhesion is stronger for the loop polymer than for the straight polymer because the glass transition temperature of the loop polymer is higher than that of straight polymer. [DOI 10.1295/polymj.37.782]

KEY WORDS Coarse-grained Molecular Dynamics Simulation / OCTA / Loop Polymer / Grafted Polymer Surface /
\end{abstract}

End grafted polymers, which are polymers tethered to the substrate surface, have been extensively studied ${ }^{1-4}$ because of its importance in surface rheology such as adhesions, frictions, and lubrications. The adhesion of grafted polymer film has been studied by several groups. Leger and her coworkers ${ }^{2,5}$ have systematically studied the adhesion between the grafted polymer layer and the elastomer made by poly(dimethylsiloxane) (PDMS). They examined the adhesion force as a function of graft density, and pointed out that there is an optimum graft density to maximize the adhesion force. The separation behavior of the adhered film was studied by Creton and his coworkers. ${ }^{4,6}$ They observed the formation of cavities when the film is pulled apart in the probe-tack experiments. Klein and coworkers ${ }^{7}$ measured the surface force acting between the Langmuir-Blodgett polymer monolayers.

The adhesion of polymers has also been studied by computer simulation. Sides et al. ${ }^{8-10}$ have studied the structure of the grafted polymer film, and their interaction by large scale MD simulations, and found that there are three types in the fracture; chain pulling out, crazing, and chain scission, which are similar to those found experimentally. Morita et al. ${ }^{11}$ studied the adhesion and friction between polymer and AFM tip using MD simulations.

In this paper, we study the adhesion between endgrafted polymer films using coarse-grained molecular dynamics. We consider two types of grafted polymer (see Figure 1), the one-end grafted polymer and the (a) straight chain (b) loop chain

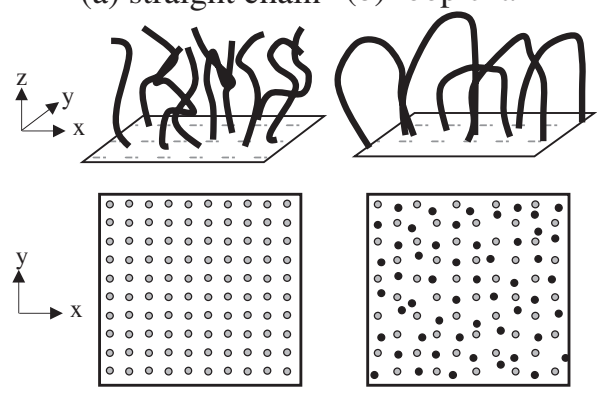

Figure 1. Schematic view of the grafted polymer film used in the present simulation. The top shows the polymers in the film, and the bottom shows the grafting point on the substrate.

two-end grafted polymer films, and call them the straight polymer (S polymer) and the loop polymer ( $\mathrm{L}$ polymer). The present study was motivated by the discussion of Klein, ${ }^{12}$ who argued that the adhesion of loop polymer will be stronger than that of straight polymer since the penetration of polymer loop into another polymer layer is expected to be less favorable than that of a single chain end. This system is much similar to the velcro tapes, and the strong adhesive force between loop chain grafted and ordinal grafted films is expected. In the molecular level, the penetration of the chain is theoretically discussed by O'Connor and McLeish. ${ }^{13}$

The present paper is constructed as follows. First we describe the property of the isolated polymer film.

${ }^{\dagger}$ To whom correspondence should be addressed (E-mail: hmorita@rheo.t.u-tokyo.ac.jp). 
Next, we discuss the loading and the unloading behavior in the adhesion between two polymer films. We shall then compare the adhesion strength of straight polymer and the loop polymer.

\section{MODEL}

For the model of the grafted polymer, we used the bead spring model. ${ }^{14}$ These model simulations are performed using the MD program COGNAC in the OCTA system. ${ }^{15,16}$ The time evolution of the bead position $\boldsymbol{r}_{n}$ was calculated by the Langevin equation, ${ }^{17,18}$

$$
m \frac{\mathrm{d}^{2} \boldsymbol{r}_{n}}{\mathrm{~d} t^{2}}=-\frac{\partial U\left(\left\{\boldsymbol{r}_{n}\right\}\right)}{\partial \boldsymbol{r}_{n}}-\Gamma \frac{\mathrm{d} \boldsymbol{r}_{n}}{\mathrm{~d} t}+\mathbf{W}_{n}(t),
$$

where $m$ is the mass of bead, $U$ is the total potential energy of the system, $\Gamma$ is the friction constant, and
$\mathbf{W}_{n}(t)$ is a Gaussian white noise which is generated according to following equation.

$$
\left\langle\mathbf{W}_{n}(t) \mathbf{W}_{m}\left(t^{\prime}\right)\right\rangle=2 k_{\mathrm{B}} T m \Gamma \delta_{n m} \mathbf{I} \delta\left(t-t^{\prime}\right)
$$

The potential energy $U\left(\left\{\boldsymbol{r}_{n}\right\}\right)$ consists of three terms, the bond potential, the bead-bead potential, and the bead-wall potential:

$$
\begin{aligned}
U\left(\left\{\boldsymbol{r}_{n}\right\}\right)= & U^{\text {bond }}\left(\left\{\boldsymbol{r}_{n}\right\}\right)+U^{\text {bead-bead }}\left(\left\{\boldsymbol{r}_{n}\right\}\right) \\
& +U^{\text {bead-wall }}\left(\left\{\boldsymbol{r}_{n}\right\}\right)
\end{aligned}
$$

The bead-bead potential is given by

$$
U^{\text {bead-bead }}\left(\left\{\boldsymbol{r}_{n}\right\}\right)=\sum_{n<m} U^{\mathrm{LJ}}\left(r_{n m}\right)
$$

where $r_{n m}=\left|\boldsymbol{r}_{n}-\boldsymbol{r}_{m}\right|$ is the distance between the bead $n$ and $m$, and $U^{\mathrm{LJ}}(r)$ is the truncated LenardJones potential given by

$$
U^{\mathrm{LJ}}(r)= \begin{cases}4 \varepsilon\left[\left\{\left(\frac{\sigma}{r}\right)^{12}-\left(\frac{\sigma}{r}\right)^{6}\right\}-\left\{\left(\frac{\sigma}{r_{\mathrm{cut}}}\right)^{12}-\left(\frac{\sigma}{r_{\mathrm{cut}}}\right)^{6}\right\}\right], & \left(r \leq r_{\mathrm{cut}}\right) \\ 0, & \left(r>r_{\mathrm{cut}}\right)\end{cases}
$$

$r_{\text {cut }}$ was chosen to be $5 \sigma$. The bead-wall potential is given by

$$
U^{\text {bead-wall }}\left(\left\{\boldsymbol{r}_{n}\right\}\right)=\sum_{n} U^{\text {Wall }}\left(z_{n}\right)
$$

where $z_{n}$ is the $z$ coordinate of the bead $n$ (the wall is assumed to be placed in the $x-y$ plane), and $U^{\text {Wall }}(z)$ is given by

$$
U^{\text {Wall }}(z)=4 \varepsilon_{w}\left[\frac{1}{5}\left(\frac{\sigma_{w}}{z}\right)^{10}-\frac{1}{2}\left(\frac{\sigma_{w}}{z}\right)^{4}\right]
$$

In eq 6 , the cut off distance, which is used in eq 4 , is not included.

This bead-wall interaction potential $U^{\text {Wall }}(z)$ was derived by the integral of $U^{\mathrm{LJ}}(r)$ for the wall atoms uniformly distributed in the wall. Finally the bonding potential is given by

$$
U^{\text {bond }}\left(\left\{\boldsymbol{r}_{n}\right\}\right)=\sum_{i} U^{\mathrm{B}}\left(r_{i}^{\mathrm{B}}\right),
$$

where $r_{i}^{\mathrm{B}}$ is the length of the $i$-th bond which is connected to the bead $n$, and $U^{\mathrm{B}}(r)$ is given by the FENE-Lennard-Jones potential:

with

$$
U^{\mathrm{B}}(r)=U^{\mathrm{FENE}}(r)+U^{\mathrm{LJ}}(r) .
$$

$$
U^{\mathrm{FENE}}(r)= \begin{cases}-\frac{1}{2} k R_{0}^{2} \ln \left(1-\left(\frac{r}{R_{0}}\right)^{2}\right), & \left(r \leq R_{0}\right) \\ \infty, & \left(r>R_{0}\right)\end{cases}
$$

where $k$ is the spring constant, and $R_{0}$ is the maximum extension of the spring. The parameters are taken as $k=30.0 \varepsilon / \sigma^{2}, R_{0}=3.0 \sigma$.

The film of the straight polymer involves 100 chains, each consisting of 40 beads. The graft points are placed regularly on the square lattice with the neighboring distance $\ell_{\mathrm{S}}=2.66 \sigma$, where $\sigma$ is the bead diameter (see eq 4). This corresponds to the weakly stretched brush. On the other hand, the film of loop polymer involves 50 chains, each consisting of 80 beads. One end of the loop polymer are fixed regularly on the square lattice with the neighboring distance $\ell_{\mathrm{L}}=\sqrt{2} \ell_{\mathrm{S}}=3.76 \sigma$, and the other ends are fixed at the position chosen randomly on the circle of radius $10 \sigma$ centered on the first end.

We took $m, \sigma$ and $\varepsilon$ as the unit of mass, length and energy respectively. The unit of time $\tau$, and the unit of the temperature $T_{0}$ are defined by

$$
\tau=\sigma(m / \varepsilon)^{1 / 2}, \quad T_{0}=\varepsilon / k_{\mathrm{B}} .
$$

The temperature $T$ was changed in the range $0.2\left[T_{0}\right]<T<2.0\left[T_{0}\right]$ and $\Gamma$ is taken to be $0.5\left[\tau^{-1}\right]$. The parameters characterizing the wall potential, $\varepsilon_{\mathrm{w}}$ and $\sigma_{\mathrm{w}}$ are taken to be $\varepsilon_{\mathrm{w}}=\varepsilon$ and $\sigma_{\mathrm{w}}=\sigma$.

To get the equilibrium structure of the film at given temperature, we started from the completely stretched configuration of the grafted chains at very high temperature $\left(T=4.0\left[T_{0}\right]\right)$, and decreased the temperature gradually. At each temperature, we performed the simulation for 500,000 time steps to relax the system, and then continued the simulation for another 500,000 time steps to get the statistical data. 


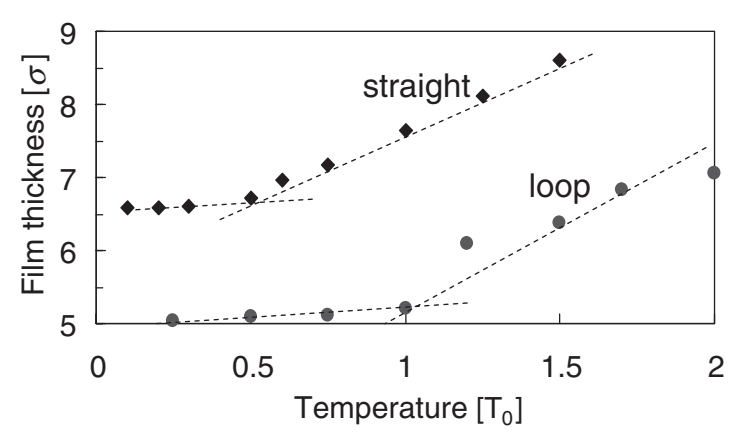

Figure 2. The film thickness of grafted polymer is plotted against the temperature.

\section{RESULTS}

\section{Glass Transition Temperature of Grafted Polymer Film}

First we discuss the glass transition temperature $T_{\mathrm{g}}$ of our film. In the bulk system, the glass transition temperature is estimated by the plot of the specific volume, or density, against the temperature. In the film of grafted polymer, the density is not uniform across the film. To estimate the glass transition temperature, we plotted the film thickness $H$ against the temperature. We defined the film thickness $H$ by twice of the $z$ coordinate of the center of mass:

$$
H=2\left\langle\frac{1}{\mathrm{~N}} \sum_{n} z_{n}\right\rangle
$$

Figure 2 shows the plot of $H$ against the temperature. It is seen that $H$ decreases with the decrease of temperature, and that the slope of the curves changes at a certain temperature: $T=0.5\left[T_{0}\right]$ for straight polymer and $T=1.0\left[T_{0}\right]$ for loop polymer. These temperatures are considered to correspond to the glass transition temperature.

To confirm that the temperatures obtained above correspond to the glass transition temperature, we calculated the mean square displacement of beads in the time interval $\Delta t$ :

$$
\left\langle(\boldsymbol{r}(\Delta t)-\boldsymbol{r}(0))^{2}\right\rangle=\frac{1}{\mathrm{~N}} \sum\left\langle\left(\boldsymbol{r}_{n}(\Delta t)-\boldsymbol{r}_{n}(0)\right)^{2}\right\rangle
$$

The time $\Delta t$ was taken to be $25 \tau$. This time is much longer than the mean collision time of beads, and is much smaller than the entanglement time $\tau_{\mathrm{e}}$. Figure 3 shows the plot of $\left\langle(\boldsymbol{r}(\Delta t)-\boldsymbol{r}(0))^{2}\right\rangle$ against the temperature. It is seen that the mobility increases markedly at the temperature obtained above: at $0.5\left[T_{0}\right]$ for straight polymer and at $c a .1 .0\left[T_{0}\right]$ for loop polymer. Thus it is reasonable to regard the temperature obtained above as the glass transition temperatures.

The glass transition temperature of loop polymer is

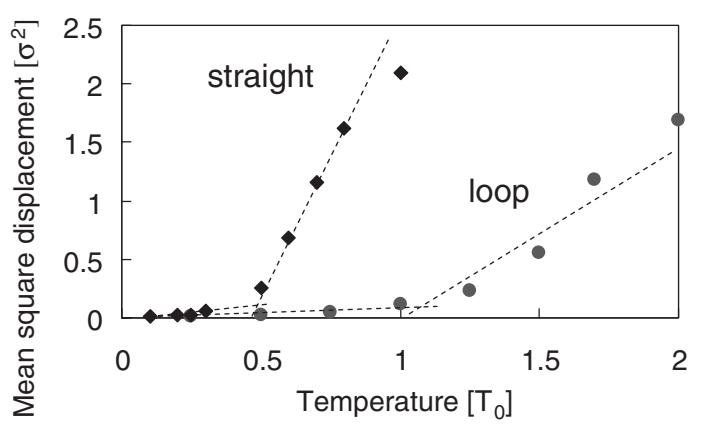

Figure 3. The mean square displacement of segments in time $25 \tau$ is plotted against the temperature.

expected to be higher than the glass transition temperature of straight polymer since the loop polymer has no chain ends which increase the free volume, and since the loop polymer are subject to extra constraints. Although Figure 2 agrees with this expectation, it is unexpected that the glass transition temperature of loop polymer is twice as high as that of straight polymer. The large difference in the glass transition temperature can be associated with the large difference in the polymer segment density: the film thickness of loop polymer is about $20 \%$ less than that of straight polymer, or the segment density in the film of loop polymer is about $20 \%$ larger than that of straight polymer.

As this stage we cannot identify the reason why the glass transition temperature or the segment density of the loop polymer is so much different from those of straight polymer. But two reasons can be considered.

A possible reason is that it is a result of the rather artificial constraint we used for the loop polymer. We imposed that the distance between the graft point is fixed at $10 \sigma$. This distance is larger than the mean end-to-end distance of Gaussian chain consisting of 80 beads. Considering that the chains are rather stretched in $z$ direction in the grafted polymer, the constraint we imposed for the loop polymer is perhaps too stringent.

The other possible reason is the entanglement effect. The entanglement effect of straight polymer is not significant for the chain length used in the present simulation. On the other hand, the loop polymers do entangle with each other by the topological constraint introduced when the loops are formed. The entanglement effect act to compress the polymers towards the wall, and can created the large difference in the segment density.

Although we do not have clear explanation for the large difference in the glass temperature for the loop polymer and straight polymer, our simulation clearly indicates that the glass transition temperatures are different for these two polymers. We believe that the present simulation reflects the reality at least 
qualitatively, even though its quantitative accuracy may be poor. This point needs to be taken into account in the comparison between the simulation and real experiments.

\section{Loading and Unloading Processes}

Next we conducted the simulation for the loading and the unloading processes in the adhesion between two polymer films. Initially we placed two films separated from each other, and then decreased the distance $\mathrm{L}_{z}$ between the two substrates at constant rate: this was done by changing by $\Delta \mathrm{L}_{z}$ by $2 \times 10^{-3} \sigma$ and displacing all beads affinely at every 20 time steps. Thus $\mathrm{dL}_{z} / \mathrm{d} t$ is $-0.01 \sigma / \tau$ in the loading process and $0.01 \sigma / \tau$ in the unloading process. The stress acting on the wall is calculated directly by calculating the total force acting on the wall:

$$
\begin{aligned}
\operatorname{Stress}\left[m / \sigma \tau^{2}\right]= & \frac{1}{S}\left(\sum_{\text {grafted beads }} F_{n, z}\right. \\
& \left.+\sum_{\neq \text {grafted beads }} F_{n, \text { wall-beads }}\right),
\end{aligned}
$$

where $S$ is the area of the wall, $F_{n, z}$ the $z$ component of the force acting on the $n$-th grafted bead, and $F_{n \text {,wall-beads }}$ is the $z$ component of the wall force acting on non-grafted beads $n$. The sign of the stress is taken to be such that it is positive when the wall is repelling to each other. The switch from loading to unloading was done when the repulsive stress becomes equal to $2.0\left[\mathrm{~m} / \sigma \tau^{2}\right]$. The loading process was stopped when the stress reaches the value $2.0\left[\mathrm{~m} / \sigma \tau^{2}\right]$, and then the unloading process was started immediately.

In the following, we report the results of two cases, the adhesion between straight-straight polymer, and the adhesion between straight-loop polymer, each are represented by SS adhesion and SL adhesion respectively. The upper film is made of straight polymer, and the lower film is made of either the straight polymer or the loop polymer. Initial configuration was chosen from one of the equilibrium configurations generated in the previous section. Simulations were done at five temperatures $T=0.25,0.5,0.75,1.0$, $1.25\left[T_{0}\right]$, which were chosen from the consideration of the glass transition temperature described in the previous section.

Figures 4, 5, and 6 show the typical stress-distance curves in the loading and the unloading processes in the SS adhesion. In the loading process, the force is first attractive due to the attractive part of the Lenard-Jones potential, but turns to be repulsive as the film is compressed strongly. The stress-distance curve in the unloading process does not follow the curve of the loading process: there is a hysteresis

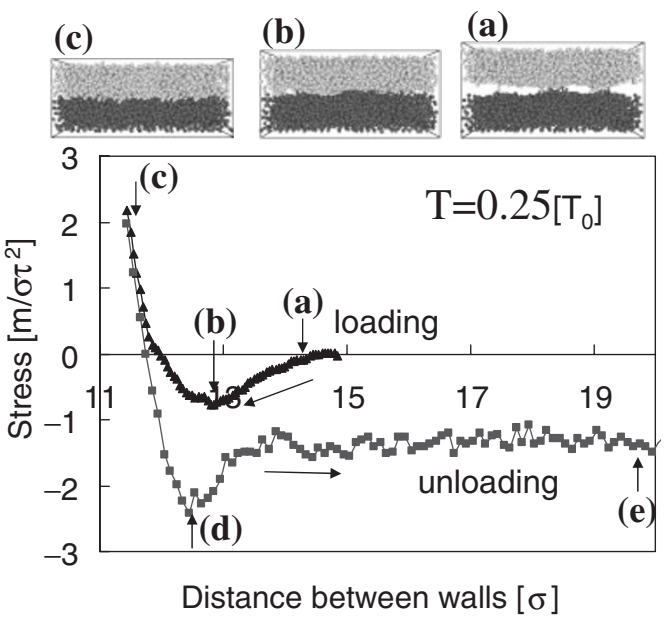

(e)

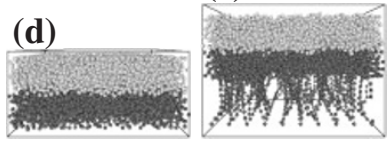

(f)

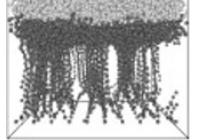

Figure 4. Stress-distance curves in the loading and the unloading processes of adhesion between straight polymer films. The attached are the snapshots of the molecular configuration in each state indicated by the arrows. The temperature is $T=$ $0.25\left[T_{0}\right]$.

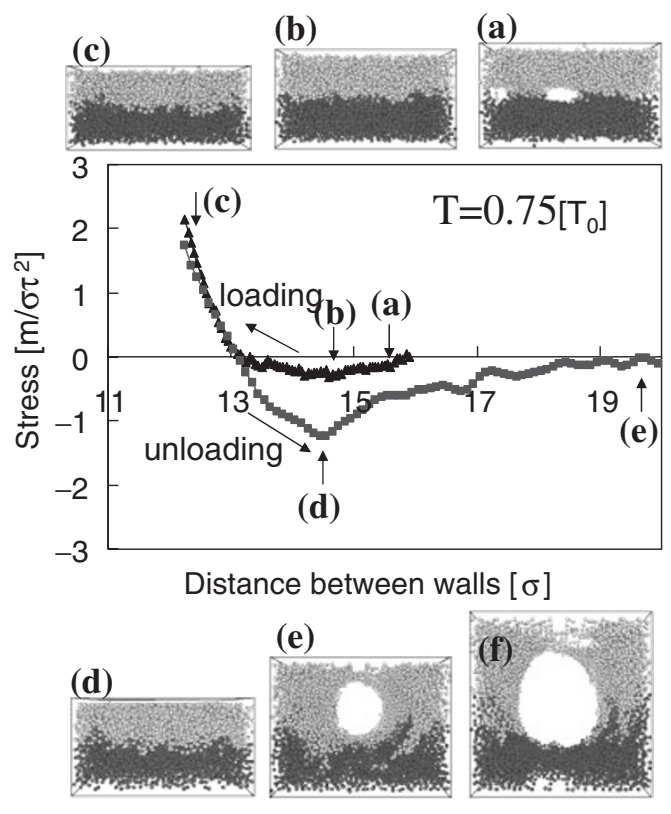

Figure 5. The same as in Figure 4. The temperature is $T=$ $0.75\left[T_{0}\right]$.

effect. In the unloading process, the behavior of the stress-distance curve varies significantly depending on the temperature. At low temperature $\left(T=0.25\left[T_{0}\right]\right)$, the attractive force shows maximum and then takes a constant value independent of separation over a large range of distance. On the other hand, at high temperature $\left(T=0.75\left[T_{0}\right]\right.$ and $\left.1.25\left[T_{0}\right]\right)$, the attractive force shows maximum and then relaxes to zero 

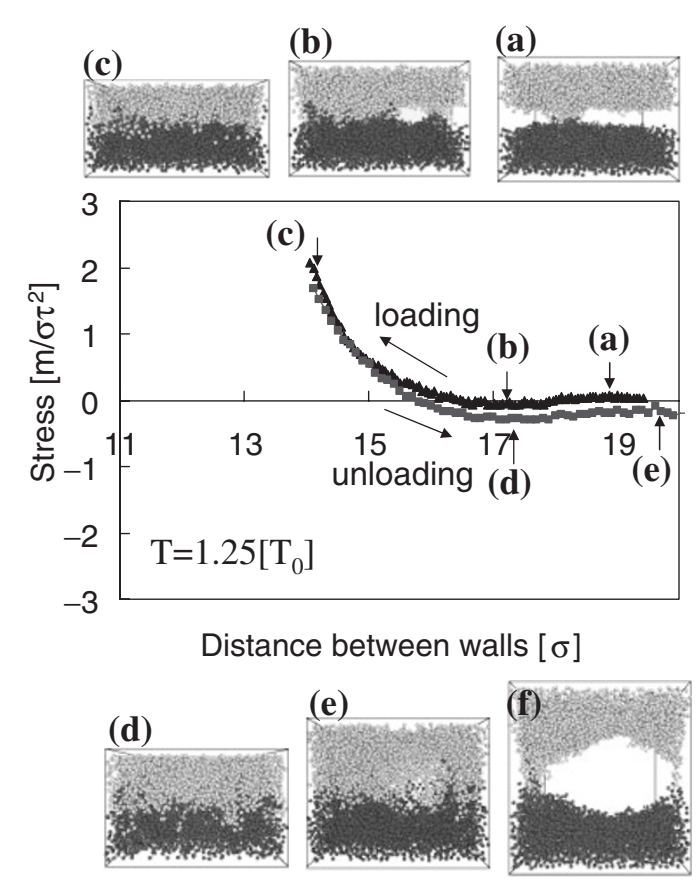

Figure 6. The same as in Figure 4. The temperature is $T=$ $1.25\left[T_{0}\right]$.

as the distance increases. These characteristic differences in the stress-distance curve in the unloading processes can be understood from the snap shots of the molecular configuration attached to Figures 4-6.

At low temperature $\left(T=0.25\left[T_{0}\right]\right)$, we see fibrils formed near the bottom layer. The fibrils are made of chains which are pulled out from the bulk region (i.e., the middle region). Since the tension in the chain is almost independent of how much it is pulled out from bulk, the stress is independent of the distance $\mathrm{L}_{z}$. We shall call such mode fibril separation.

Figure 7 shows the zoom up of the fibril structure. Here the surface at which the segment density becomes less than 0.3 is shown. The figure also shows the mean stretch of the bond calculated at each layer in the film. The bond length is uniform in most cases, but becomes non uniform when the fibrils are made.

At higher temperature $\left(T=0.75\left[T_{0}\right]\right.$ and $\left.1.25\left[T_{0}\right]\right)$, we see cavities rather than fibrils. The cavities first appear near the surface of the wall where the segment density is low. As the distance $\mathrm{L}_{z}$ increases, the cavities moves towards the center, i.e., the intersection between the two polymer films and then merges and grows, eventually separating the two films. We shall call such separation interfacial separation. Figure 8 shows the close up of the cavity structure. It is seen that the bond length remains rather uniform even when cavities are formed. This indicates that the tension along the chain is relaxed at high temperature.

\section{Adhesion Strength}

Having discussed the mode of separation, we now
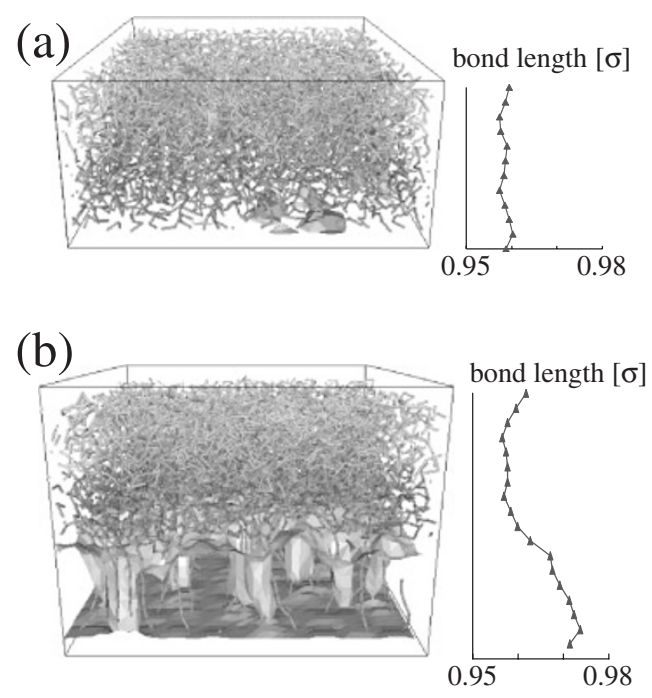

Figure 7. Zoom up of the molecular configuration at temperature $T=0.25\left[T_{0}\right]$, at the separation of The numbers on the right hand side shows the average of the strain of the bond length $\Delta \ell / \ell$ calculated in the layer of thickness $0.5 \sigma$.

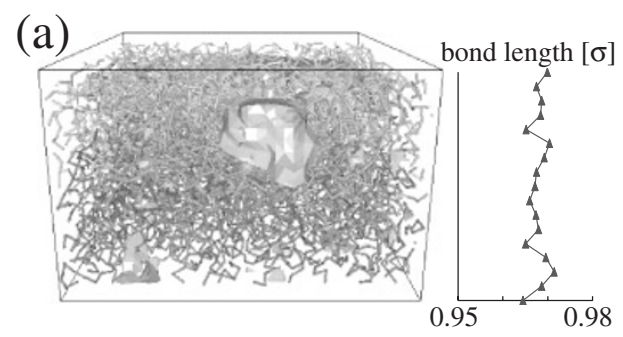

(b)

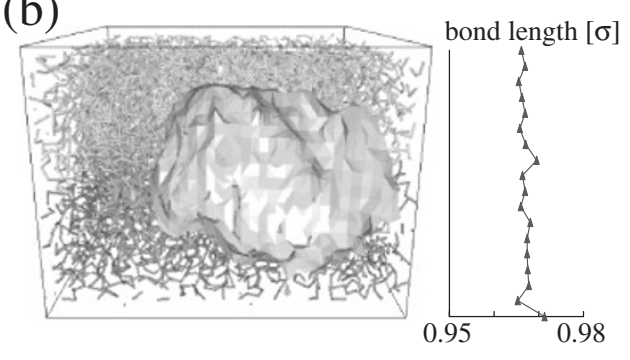

Figure 8. Zoom up of the molecular configuration at temperature $T=1.25\left[T_{0}\right]$, at the separation of The numbers on the right hand side shows the average of the strain of the bond length $\Delta \ell / \ell$ calculated in the layer of thickness $0.5 \sigma$.

discuss the adhesion strength. We define the adhesion strength by a maximum attractive stress in the unloading process. Figure 9 shows the temperature dependence of the adhesion strength. As the temperature increases, the adhesion strength decreases. The adhesion strength of a loop polymer is always larger than that of straight polymer.

Two reasons can be considered for the reason why the SL adhesion is stronger than the SS adhesion. One is the entanglement effect, and the other is the effect of the mobility difference (or the difference of the 


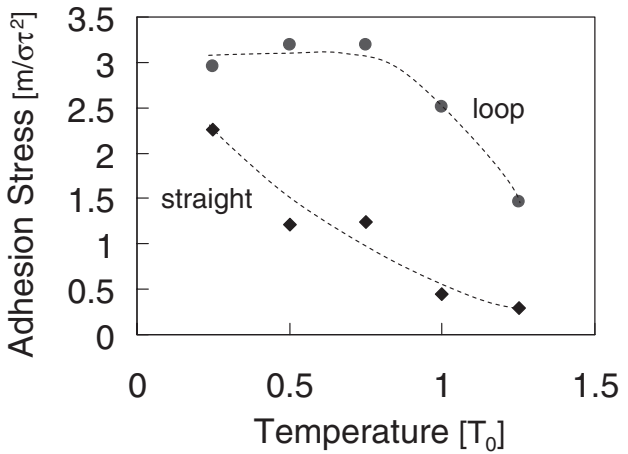

Figure 9. Adhesion strength (the maximum attractive stress in the unloading process) is plotted against the temperature for the straight-straight adhesion, and the straight-loop adhesion.

glass transition temperature). We consider that in the particular case studied here it is mainly the effect of mobility difference. The reason is as follows.

(1) The chain considered here is not long enough for the entanglement effect to be important. The entanglement length of the present model in the bulk is estimated to be $35,{ }^{18}$ which is about the same as the chain length of the straight polymer in the present simulation. Since the entanglement in the grafted chain is considered to take place less frequently than in the bulk, it is unlikely that the entanglement effect plays the key role in the present system.

(2) The speed of loading and unloading is much faster than the formation of entanglement, and it is unlikely that effective entanglement is formed in the loading process. Therefore we think the difference in the adhesion strength in the present system is due to the mobility difference.

Figure 10 shows the molecular configuration when the films are going to separate from each other in the unloading process. At low temperature $\left(T=0.25\left[T_{0}\right]\right)$, the fibril separation takes place. At $T=0.5\left[T_{0}\right]$, the separation turns into interfacial type in the SS adhesion, while the separation is still in fibril type in SL adhesion. This can be understood as follows.

As the films are pulled apart, the cavities are elongated. If the system has enough mobility, the cavities merge together to form larger spherical cavity. On the other hand, if the system has low mobility, the merging of the cavities is suppressed, and the cavities are elongated to form fibrils. In the case of SS adhesion, the transition takes place at $T=0.5\left[T_{0}\right]$ where the both polymer films starts to have large mobility. In the case of SL adhesion, this transition cannot take place since the loop polymer is glassy phase and the straight polymer is constrained by the loop polymers. In the example shown in Figure 10, the fibril are seen in both films at $T=0.75\left[T_{0}\right]$. (The phenomenon that fibrils are formed in the two films is also seen in the

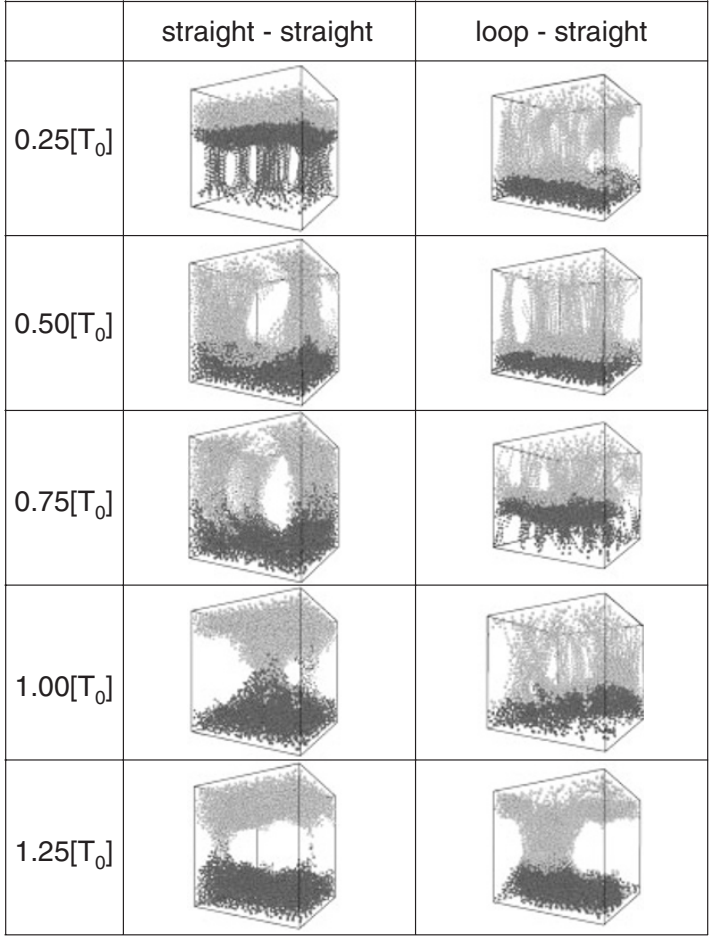

Figure 10. Chain configurations when the films are separated from each other in the unloading process at various temperatures shown on the left. The figure shows the chain configuration when the substrates are separated by the distance $15 \sigma$ after the start of unloading.

SS adhesion.) The cavities are seen only when the temperature becomes above the glass transition of the loop polymer $\left(T=1.0\left[T_{0}\right], 1.25\left[T_{0}\right]\right)$.

We think that the cavities and fibrils seen in our simulation correspond to those which have been observed in real experiments although the scales are considerably different: the cavities seen by Creton et $a l$. have size of the order of few tens $\mu \mathrm{m}$, while the cavity size of our simulation is of the order of $\mathrm{nm}$. Thus the cavity in our simulation corresponds to the micro cavities at the very early stage of cavity formation. Likewise, the fibrils in our simulations correspond to micro fibrils. Although their sizes are different, we believe that they share many common aspects in their structure and the mechanisms of formation.

We therefore think that the difference in the adhesion strength is due to the difference in the separation mode: in the case of SL adhesion, the fibrils are formed, which gives larger adhesion strength than that of the SS adhesion.

The above consideration indicates that the glass transition temperature is the key parameter in the adhesion strength. To confirm this, we have plotted the adhesion force against the reduced temperature $\left(T-T_{\mathrm{g}}\right) / T_{\mathrm{g}}$ in Figure 11. It is seen that in this plot, the curve of the loop polymer comes close to that of straight polymer. 


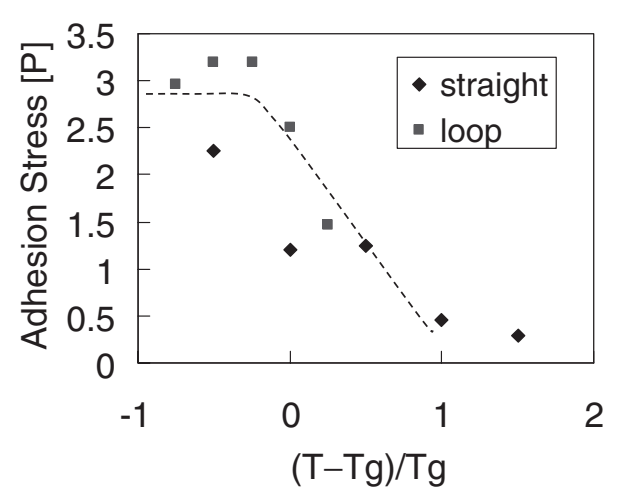

Figure 11. Adhesion strength is plotted against the reduced temperature $\left(T-T_{\mathrm{g}}\right) / T_{\mathrm{g}}$ for the straight-straight adhesion, and the straight-loop adhesion.

\section{CONCLUSIONS}

In this paper, we have shown the results of the molecular dynamics study of the adhesion between grafted polymer films of two types of polymer, the straight polymer and the loop polymer. We have shown that the adhesion behavior is strongly dependent on the mobility of the polymer film. The separation mode changes from fibril separation to interfacial separation as the temperature increases.

Our simulation indicates that the adhesion becomes stronger if one of the films is replaced by the loop polymer. In the present system studied here, we have shown that this is because the glass transition temperature of the loop polymer is higher than that of the straight polymers. Clearly this conclusion is not general: the result will be different for films made of larger polymers, where the entanglement effect will play an important role in the adhesion. Our study indicates that the glass transition temperature can be the key factor in determining the adhesion strength.
Acknowledgment. The authors thank T. Yamaue, M. Makino, and E. Nishitani for many helpful comments and discussions. We also thank the reviewer who suggested the plot of the adhesion strength against the reduced temperature. This work is supported by The Japan Science and Technology Agency (JST).

\section{REFERENCES}

1. R. P. Wool, "Polymer Interfaces: Structure and Strength," Hanser, Munich, 1995.

2. L. Leger, E. Raphael, and H. Hervet, Adv. Polym. Sci., 138, 185 (1999).

3. G. Grest, Adv. Polym. Sci., 138, 149 (1999).

4. C. Creton, E. J. Kramer, H. R. Brown, and C.-Y. Hui, Adv. Polym. Sci., 156, 53 (2001).

5. M. Deruelle, L. Leger, and M. Tirrell, Macromolecules, 28, 7419 (1995).

6. A. C. Costaa, A. Chicheb, P. Vlcek, C. Creton, and R. J. Composto, Polymer, 45, 4445 (2004).

7. L. A. Tsarkova, P. V. Protsenko, and J. Klein, Colloid J., 66, 84 (2004).

8. S. W. Sides, G. S. Grest, M. J. Stevens, and S. J. Plimpton, J. Polym. Sci., Part B: Polym. Phys., 42, 199 (2004).

9. S. W. Sides, G. S. Grest, and M. J. Stevens, Macromolecules, 35, 566 (2002).

10. S. W. Sides, G. S. Grest, and M. J. Stevens, Phys. Rev. E: Stat. Phys., Plasmas, 64, 050802 (2001).

11. H. Morita, T. Ikehara, T. Nishi, and M. Doi, Polym. J., 36, 265 (2004).

12. J. Klein, J. Phys.: Condens. Matter, 12, A19 (2000).

13. K. P. O'Connor and T. C. B. McLeish, Macromolecules, 26, 7322 (1993).

14. M. Murat and G. S. Grest, Macromolecules, 22, 4054 (1989).

15. http://octa.jp

16. T. Aoyagi, F. Sawa, T. Shoji, H. Fukunaga, J. Takimoto, and M. Doi, Comput. Phys. Commun., 145, 267 (2002).

17. G. S. Grest and K. Kremer, Phys. Rev. A: At., Mol., Opt. Phys., 33, 3628 (1986).

18. K. Kremer and G. S. Grest, J. Chem. Phys., 92, 5057 (1990). 